\title{
VeChat: Correcting errors in long reads using variation graphs
}

\author{
Xiao Luo ${ }^{1,2}$, Xiongbin Kang ${ }^{1}$, Alexander Schönhuth ${ }^{1,2,{ }^{*}}$ \\ ${ }^{1}$ Genome Data Science, Faculty of Technology, Bielefeld University, Bielefeld, Germany \\ ${ }^{2}$ Life Science \& Health, Centrum Wiskunde \& Informatica, Amsterdam, The Netherlands \\ * To whom correspondence should be addressed.
}

Email: a.schoenhuth@cwi.nl

\begin{abstract}
Error correction is the canonical first step in long-read sequencing data analysis. The current standard is to make use of a consensus sequence as a template. However, in mixed samples, such as metagenomes or organisms of higher ploidy, consensus induced biases can mask true variants affecting haplotypes of lower frequencies, because they are mistaken as errors.

The novelty presented here is to use graph based, instead of sequence based consensus as a template for identifying errors. The advantage is that graph based reference systems also capture variants of lower frequencies, so do not mistakenly mask them as errors. We present VeChat, as a novel approach to implement this idea: VeChat distinguishes errors from haplotype-specific true variants based on variation graphs, which reflect a popular type of data structure for pangenome reference systems. Upon initial construction of an ad-hoc variation graph from the raw input reads, nodes and edges that are due to errors are pruned from that graph by way of an iterative procedure that is based on principles from frequent itemset mining. Upon termination, the graph exclusively contains nodes and edges reflecting true sequential phenomena. Final re-alignments of the raw reads indicate where and how reads need to be corrected.

Extensive benchmarking experiments demonstrate that PacBio and ONT reads corrected by VeChat contain 4 to 15 , or, respectively, 2 to 10 times less errors than when corrected state of the art approaches. VeChat is implemented in an easy-to-use open-source tool and publicly available at https://github. com/HaploKit/vechat.
\end{abstract}

\section{Keywords}

Error correction; Long reads; Haplotype; Polyploid; Metagenome

\section{Background}

Third generation sequencing (TGS) such as single-molecule real-time (Pacific Biosciences, or short PacBio) or nanopore sequencing (Oxford Nanopore Technologies or short ONT) has been emerging rapidly over the last few years. Beyond the obvious reason that TGS reads tend to be longer by orders of magnitude - read length ranges from several kbp up to even a few Mbp (Logsdon et al., 2020), whereas next-generation sequencing reads usually span only a few hundred base pairs - the fact that TGS is relatively inexpensive adds to its popularity. Moreover, TGS circumvents polymerase chain reaction $(\mathrm{PCR})$ as part of its protocol, which prevents related biases. Thanks to these advantages, TGS has been 
able to make decisive contributions in various areas of application. Prominent examples are haplotype phasing (Schrinner et al., 2020), genome assembly (Jain et al., 2018b; Ruan and Li, 2020; Shafin et al., 2020; Kolmogorov et al., 2020; Miga et al., 2020) and (complex) variant calling (Edge and Bansal, 2019; Thibodeau et al., 2020; Fujimoto et al., 2021).

On the other hand, however, the downside of TGS are the significantly elevated error rates the reads are affected with. For example, PacBio CLR and ONT reads, as the currently most representative examples of TGS reads, contain 5\% to 15\% errors (Logsdon et al., 2020). This comes in obvious contrast to NGS short reads, whose error rates usually do not exceed 1\%. The fact that the majority of errors affecting long reads consists of insertions and deletions adds to the difficulties because it prevents the application of principles and straightforward adaptation of tools for correcting errors in short reads. At any rate, direct usage of raw TGS reads is hardly possible in the majority of relevant applications. So, novel methods and tools are required.

Because correcting errors in TGS reads is imperative for sound analyses and because correcting errors in TGS reads is a must, various TGS read error correction methods have been presented in the meantime. The corresponding range of methods can be divided into two major categories: hybrid correction and self correction. While hybrid correction addresses to integrate short reads in the process, self correction seeks to correct errors without auxiliary data.

Clearly, hybrid correction reflects a sound and reasonable approach in general (see (Hackl et al., 2014; Salmela and Rivals, 2014; Firtina et al., 2018; Morisse et al., 2018) for prominent approaches). However, hybrid correction suffers from some pragmatic issues. First, while long reads can span repetitive regions, short reads cannot; this introduces ambiguities in the process of assigning short to long reads (or vice versa), and as a consequence biases in the quality of the correction. Second, short reads re-introduce PCR induced biases. For example, certain areas of genomes are not sufficiently covered by short reads because of sequence content (e.g. GC content). This hampers error correction in these areas. Last but not least, sequencing genome samples employing several different protocols can be entirely impossible, which prevents the application of hybrid error correction in the first place.

Self-correction, as the second class of methods, does not suffer from any of these issues. However, because of the lack of external (e.g. short read based) assistance, self-correction faces other methodically principled challenges. It is key to overcome these challenges before one can profit from the great practical advantages of self error correction.

In terms of prior, related work, self-correction can be further divided into three sub-categories, each of which is characterized by particular algorithmic strategies and methodical foundations.

The first, and most common of the three categories is based on multiple sequence alignments (MSAs). For prior approaches and tools that crucially rely on computing MSAs, see Racon (Vaser et al., 2017), the error correction module of the assembler Canu (Koren et al., 2017), and FLAS (Bao et al., 2019).

The second principled class of approaches relies on de Bruijn graphs (DBGs). Corresponding tools employ DBGs at some point crucial for the correction process. The prevalent tool to consider is Daccord (Tischler and Myers, 2017), which is based on raising local DBGs, where local refers to DBGs reflecting relatively small segments of the genome the reads stem from.

The third class of self correction methods collects approaches that make combined use of both MSAs and DBGs during the process. Such methodically combined approaches seek to balance the advantages and disadvantages of the two concepts, MSAs on the one hand, and DBGs on the other hand. Prominent tools that make successful, combined use of MSAs with DBGs are LoRMA (Salmela et al., 2017) and CONSENT (Morisse et al., 2021).

The common denominator that unifies all of these approaches is to raise consensus sequence that reflects a summary of the reads observed, and serves as a guideline during error correction. However, sequence-based templates cannot capture ambiguities, whcih explains the corresponding biases during the correction process: for each polymorphic site, one needs to decide on one of the possible alleles while discarding all others. As a consequence, these approaches tend to mask variation that characterizes little covered haplotypes or strains in mixed samples (metagenomes, cancer genomes) or polyploid genomes. Because the haplotypes affected virtually disappear, downstream analyses remain blind with respect to them. 
To address this issue, we developed VeChat, a self-correction method to perform haplotype-aware error correction for long reads. From a larger perspective, VeChat considers the full spectrum of all possible haplotypes that possibly affect the sample already during error correction, and not only thereafter. This reflects a novelty for ploidies larger than two, because earlier approaches only deal with diploid scenarios (Luo et al., 2021). From a methodical point of view, the novelty of VeChat is to integrate variation graphs as a fundamental data structure into the process of error correction. To the best of our knowledge, VeChat is the first approach to do that.

We have tested VeChat and extensively compared it with the current state of the art on datasets reflecting various settings of current interest. Benchmarking experiments on both simulated and real data demonstrate that our approach achieves the best performance rates, across all categories of common sequencing errors.

\section{Results}

We have designed and implemented VeChat ([V]ariation graph based [e]rror [C]orrection in [ha]plo[t]ypes), a new approach to haplotype aware long read self error correction. The key concept underlying VeChat are variation graphs. Unlike single consensus sequences, which current approaches are generally centering on, variation graphs are able to represent the genetic diversity across multiple, evolutionarily or environmentally coherent genomes. This enables to preserve haplotype specific variation during error correction also for samples of higher, known or unknown ploidy.

In this section, we first provide a high-level description of the workflow of VeChat. We then evaluate the performance of VeChat on both simulated and real data in comparison with the state of the art approaches.

\section{Workflow}

See Figure 1 for an illustration of the workflow of VeChat. VeChat consists of two cycles. While the first cycle yields pre-corrected reads, the second cycle generates the final, corrected reads from the pre-corrected reads. Each cycle proceeds in 6 steps. While the two cycles generally agree on these 6 steps, they disagree in terms of crucial details affecting steps 1 and 4 .

During the first cycle, step 1 computes minimizer based all-vs-all overlaps, for which we employ Minimap2 (Li, 2018). Because using Minimap2 prevents the need for computing base-level alignments, this stage proceeds rapidly and without any further efforts. 
Steps 2-6 reflect the technical core of the error correction procedure in Figure 1. In step 2, a target read is selected as the read whose errors are to be corrected, and a read alignment pile that consists of all reads that overlap it is computed. Subsequently, in step 3 , the read alignment pile is divided into small segments, where each of the segments gives rise to a window like part of the pile in step 3 ; the part of the target read in a particular window is further referred to as 'target subread'.

Subsequently, in step 4 , the error correction for target subreads is performed in each window. Step 4 is methodically more involved, because it captures the novel, variation graph based approach; see Figure 2(a) for detailed illustrations on the version of that particular step used in the first cycle. Step 4 involves the construction of a variation graph, and pruning this graph in an iterative manner ('Graph pruning' and 'Graph re-pruning' in Figure 2(a)). Pruning reflects removing spurious nodes and edges, and models nodes and edges in terms of a frequent itemset model that involves read coverage, sequencing errors and co-occurrence of characters in reads; see subsection for details. The first cycle concludes with concatenating the different 'target subreads' of one target read, which results in a pre-corrected read at full, original length. These pre-corrected reads then serve as input for the second cycle.

While the (vast) majority of errors have already been corrected during the first cycle, a few errors, to be considered statistical outliers that escape correction during iterative graph pruning during the first cycle, have resisted their correction. The second cycle is supposed to spot such outliers. The second cycle is less complex than the first cycle, because it does no longer include the statistically involved graph pruning procedure; the blue elements in Figure 1 point out the different routes along which the second cycle proceeds. Overall, as above-mentioned, the second cycle is identical with the first cycle in steps 2 , 3,5 and 6 . In step 1 , however, beyond computing all-vs-all overlaps, base-level alignment are computed, which enables haplotype aware read overlap filtration. Step 4, as shown in Figure 2 (b), then proceeds differently insofar as graph (re-)pruning is no longer part of the cycle. Instead of iterative re-pruning, which did not lead to removal of the errors that we would like to remove

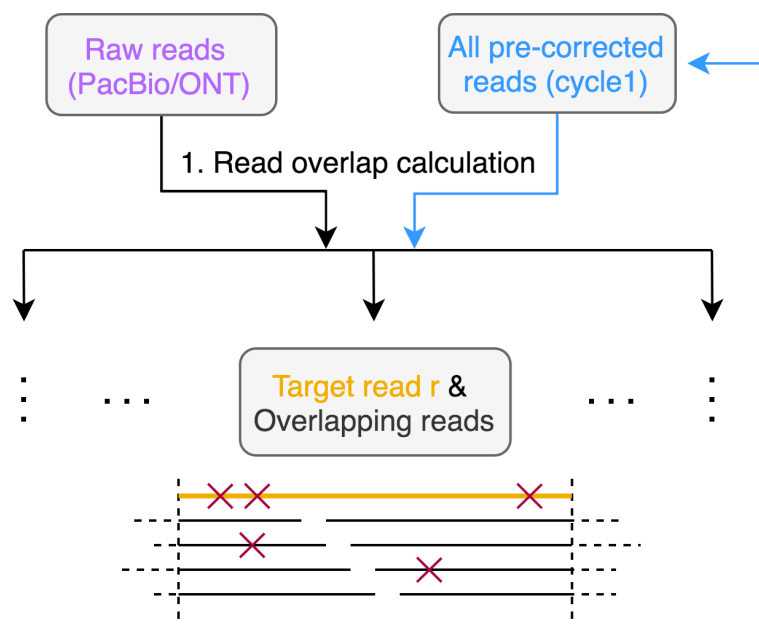

2. Read alignment pile generation
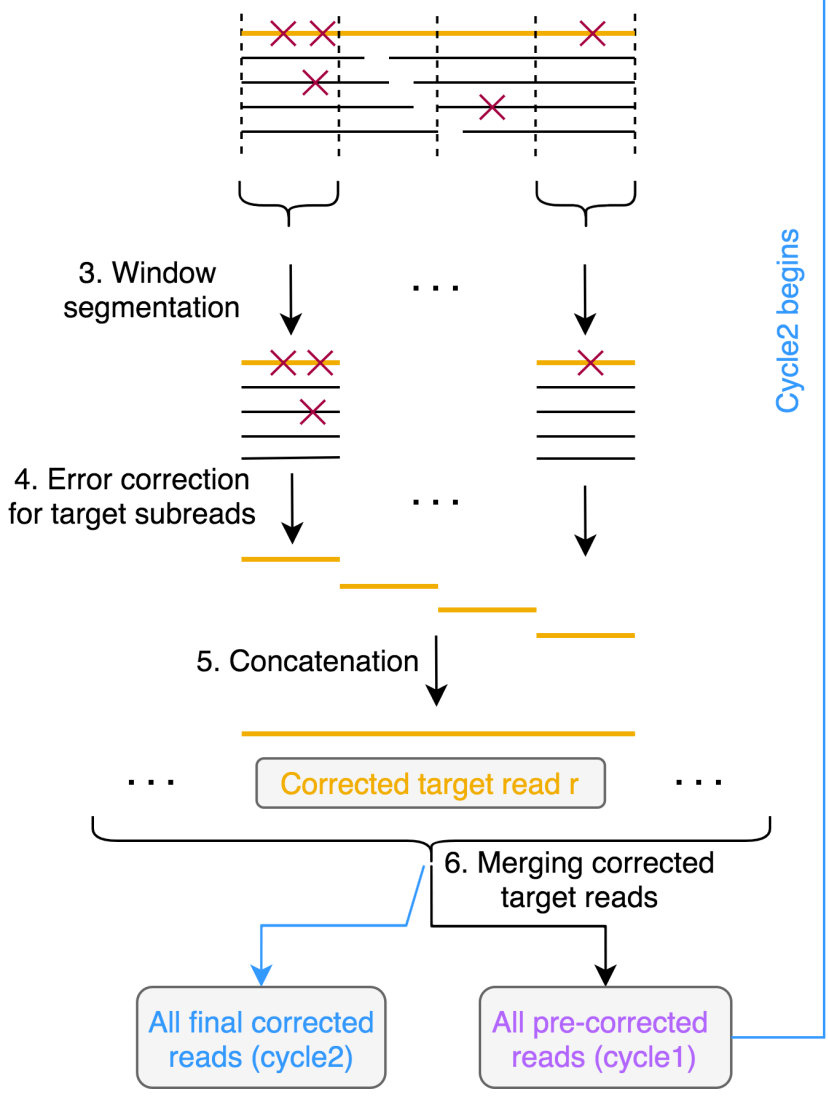

Figure 1. Workflow of VeChat. The input and output of cycle 1 and cycle 2 are labeled with purple and blue, respectively. Both cycle 1 and cycle 2 share the steps 1-6 except some differences in step 1 and 4. The target read is highlighted with orange. Red forks indicate the sequencing errors in reads. 
during this second cycle, only consensus sequences (displayed as thick yellow arrows in Figure 2(b)) are derived from the constructed variation graphs by using a dynamic programming algorithm (Lee, 2003). After concatenating the consensus sequences in step 5, joining all target reads in step 6 generates the final output of Vechat. Steps 1-6 are described in detail referring to both first and second cycle in Methods.

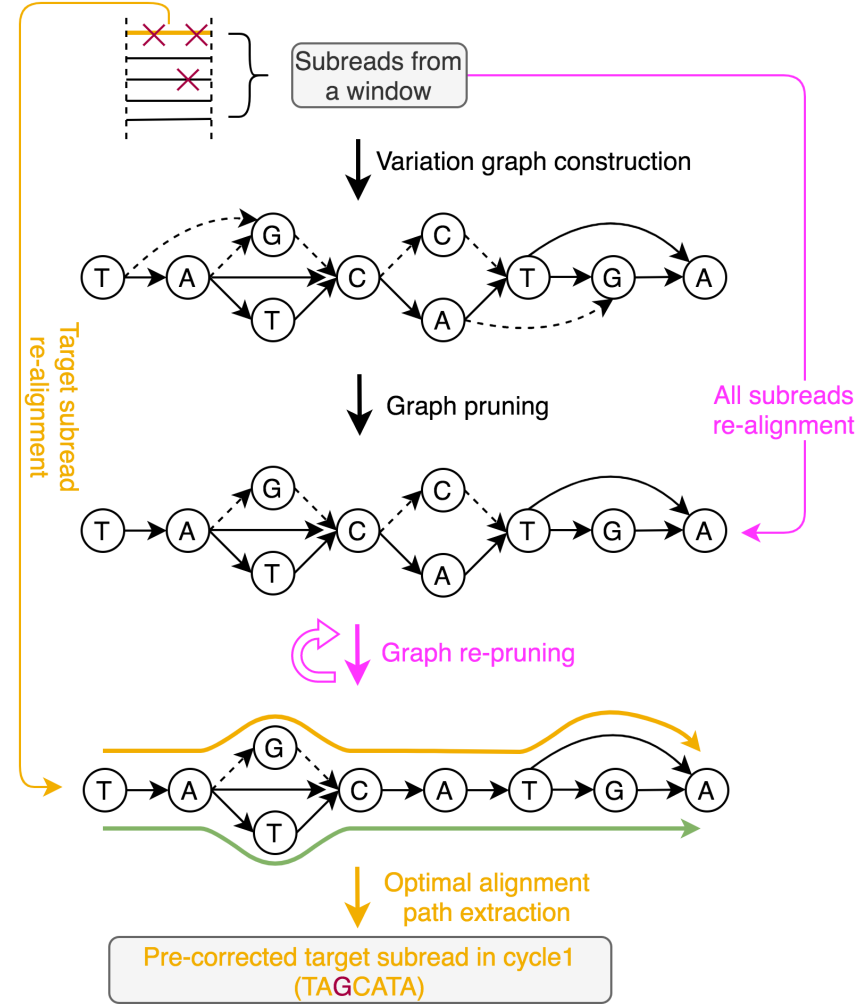

(a) Error correction for one target subread in cycle 1

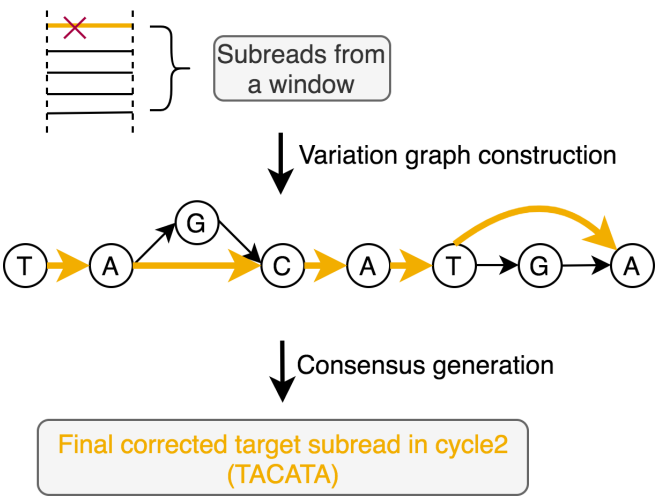

(b) Error correction for one target subread in cycle

2

Figure 2. Error correction for one target subread. (a) is the error correction diagram for one target subread in cycle 1 . The error correction process for the target read $r$ is illustrated assuming a diploid scenario (the orange path represents the optimal alignment path, whereas the green path represents the other true haplotype). "Graph pruning" and "Graph re-pruning" refer to the core error correction procedures. These procedures rely on a variation graph that is constructed from segments of a read alignment pile that results from a multiple alignment of the target read and the reads that overlap it, see Figure 1. During graph pruning and re-pruning spurious edges (dashed arrows), induced by sequencing errors, are removed from the variation graph. The pink elements indicate that these procedures are repeated. (b) is the error correction diagram for one target subread in cycle 2 . The bold orange path indicates the consensus sequence. The false nucleotide ' $G$ ' in the pre-corrected target subread in cycle 1 (see in (a)) is marked with red and further corrected in cycle 2.

\section{Datasets}

\section{Simulated sequencing data}

We made use of a very recent tool PBSIM2 (Ono et al., 2021) to simulate PacBio CLR and Oxford Nanopore reads using built-in P6C4 and R103 model-based simulation profiles, respectively. Since the main application scenario of VeChat is to correct long-read sequencing data from multiple genomes, such as polyploid genomes and metagenomes, we simulated various datasets for both cases. 
Polyploid genome data. We constructed pseudo diploid (ploidy=2, ANI: 98\%), triploid (ploidy=3, ANI: $96 \% \sim 98 \%$ ) and tetraploid (ploidy=4, ANI: $96 \% \sim 99 \%$ ) genomes by mixing strains of Escherichia coli (E. coli) bacteria; note that Average Nucleotide Identity (ANI) is defined to measure the genome sequence similarity, which can be reported by FastANI (Jain et al., 2018a), for example. All genome sequences of E. coli were downloaded from the NCBI database (see Supplementary Table S1 for the details of reference genomes). Reads were simulated from the haplotypes (i.e. strains) independently and upon generation mixed together to form the corresponding polyploid genome data sets (ploidy=2,3,4). We simulated both PacBio CLR and Nanopore reads for these datasets, at average sequencing coverage of 30x per haplotype and average sequencing error rate of $10 \%$.

Metagenome data. Additionally, we used CAMISIM (Fritz et al., 2019) to simulate two metagenomic datasets (PacBio CLR reads) of different levels of complexity. Here, we used PBSIM2 to simulate PacBio CLR reads instead of the built-in simulator in CAMISIM. The low complexity dataset consists of 10 species (20 strains), whereas the high complexity dataset consists of 30 species (100 strains). The genomes used in both datasets are derived from (Quince et al., 2017) (see Supplementary Table S1 for the details). For both datasets, the average sequencing coverage of strains is about 30x and the average sequencing error rate is $10 \%$. The relative abundances of strains range from $1.9 \%$ to $10.6 \%$ and from $0.28 \%$ to $3.3 \%$, respectively.

\section{Real sequencing data (Mock communities)}

Yeast pseudo-diploid genome data. We constructed a pseudo-diploid genome by mixing two yeast strains (N44, CBS432) of ANI 98.4\%, which are derived from Yeast Population Reference Panel (see Supplementary Table S1). The corresponding real PacBio CLR reads were downloaded from European Nucleotide Archive (ENA) under project PRJEB7245, and we only subsampled long reads with sequencing coverage of 30x per strain for further analyses.

NWC Metagenome data. We downloaded two real metagenomic datasets (PacBio CLR reads) derived from natural whey starter cultures (NWCs) (Somerville et al., 2019) and mixed both together (see Supplementary Table S1), and then subsampled 20\% reads such that we obtained a low-complexity metagenomic dataset, which contains 3 species (6 strains).

Microbial 10-plex Metagenome data. We downloaded raw long-read sequencing data and the corresponding reference genomes from a 10-plex multiplexed dataset which was sequenced by PacBio Sequel System, Chemistry v3.0 (https://downloads.pacbcloud.com/public/dataset/microbial_ multiplex_dataset_release_SMRT_Link_v6.0.0_with_Express_2.0/). Then, we randomly subsampled $10 \%$ reads such that we obtained a mock metagenomic dataset with an average sequencing coverage about 40x, which contains 7 species (9 strains in all, ANI $<98.5 \%$, see Supplementary Table S1).

\section{Benchmarking: Alternative Approaches}

To enable a fair and meaningful comparison, we considered all popular state-of-the-art tools that perform TGS read self-correction. Namely, this selection includes Racon (v1.4.13) (Vaser et al., 2017), CONSENT (v2.2.2) (Morisse et al., 2021), Canu (v2.1.1) (Koren et al., 2017) and Daccord (v0.0.18) (Tischler and Myers, 2017). We ran all tools using their default parameters.

\section{Metrics for evaluation}

Genome assembly performance is usually evaluated by means of several commonly used metrics, as reported by QUAST V5.1.0 (Mikheenko et al., 2018). See below for specific explanations, and see http://quast.sourceforge.net/docs/manual.html for full details. Note that the principled qualities of error-corrected long reads are covered by standard QUAST criteria as well; for example, low haplotype coverage reflects that true variants were mistakenly identified as errors, while error rates and misassemblies 
reflect that the correction procedure overlooked errors, or even confounded reads in terms of their origin through mistaken correction. As usual, corrected reads of length less than 500bp were filtered from the output before evaluation. Note that we ran QUAST with the option--ambiguity-usage one, which appropriately takes into account that our data sets reflect mixed samples (such as polyploid genomes or metagenomes).

Error rate (ER). The error rate is equal to the sum of mismatch rate and indel rate when mapping the obtained corrected reads to the reference haplotype sequences.

Haplotype coverage (HC). Haplotype coverage is the percentage of aligned bases in the ground truth haplotypes covered by corrected reads, which is used to measure the completeness of the corrected reads.

N50 and NGA50. N50 is defined as the length for which the collection of all corrected reads of that length or longer covers at least half the given sequences. NGA50 is similar to N50 but can only be calculated when the reference genome is provided. NGA50 only considers the aligned blocks (after breaking reads at misassembly events and trimming all unaligned nucleotides), which is defined as the length for which the overall size of all aligned blocks of this length or longer equals at least half of the reference haplotypes. Both N50 and NGA50 are used to assess the length distribution of corrected reads. Note that this may be of relatively little interest for corrected reads. We nevertheless display corresponding results because error correction does have an influence on these statistics.

Number of misassemblies (\#Misassemblies). The misassembly event in corrected reads indicates that left and right flanking sequences align to the true haplotypes with a gap or overlap of more than $1 \mathrm{kbp}$, or align to different strands, or even align to different haplotypes or strains. Here, we report the total number of misassemblies in the given sequence data.

\section{Benchmarking results}

Simulated polyploid genome datasets: PacBio. Table 1 shows the error correction benchmarking results for simulated PacBio CLR reads from genomes of varying ploidies, namely 2,3 and 4 . VeChat achieves approximately $14 \sim 30,9 \sim 26$ and $4 \sim 11$ times lower error rates on diploid, triploid and tetraploid genomes, respectively. At the same time, it maintains better or comparable performance in terms of other aspects such as number of corrected reads, completeness (haplotype coverage), number of misassemblies and length of corrected reads (as shown by N50/NGA50). In particular, VeChat drastically outperforms other tools in terms of mismatch rate $(4 \sim 69$ times lower than others).

Simulated polyploid genome datasets: ONT. Table 2 shows the error correction benchmarking results for simulated Oxford Nanopore reads from genomes of varying ploidy, namely 2, 3 and 4 . VeChat achieves approximately $10 \sim 20,3 \sim 9$ and $2 \sim 5$ times lower error rates on diploid, triploid and tetraploid genomes, respectively, while maintaining better or comparable performance in terms of all other aspects. Just as for PacBio reads, VeChat also shows better performance in terms of mismatch rate: $2 \sim 59$ times lower than other correction tools, compared with indel rate.

Simulated metagenome datasets. Table 3 shows the error correction benchmarking results for simulated PacBio CLR reads of metagenomic datasets with different complexity. VeChat achieves approximately $6 \sim 7$ and $3 \sim 4$ times lower error rates on low and high complexity metagenomes, respectively, while maintaining comparable performance in terms of other aspects. Particularly, VeChat drastically outperforms other tools in terms of mismatch rate $(6 \sim 12$ times lower) on the low complexity dataset. 


\begin{tabular}{lcccccccc}
\hline Method & \#Seq & $\begin{array}{c}\text { Error } \\
\text { rate } \\
(\%)\end{array}$ & $\begin{array}{c}\text { Mismatch } \\
(\%)\end{array}$ & $\begin{array}{c}\text { Indel } \\
(\%)\end{array}$ & $\begin{array}{c}\text { Haplotype } \\
\text { coverage } \\
(\%)\end{array}$ & $\begin{array}{c}\text { N50 } \\
(\mathrm{bp})\end{array}$ & $\begin{array}{c}\text { NGA50 } \\
(\mathrm{bp})\end{array}$ & $\begin{array}{c}\text { \# Mis- } \\
\text { assem- } \\
\text { blies }\end{array}$ \\
\hline Ploidy=2 & & & & & & & & \\
\hline VeChat & 31958 & 0.014 & 0.006 & 0.008 & 100.0 & 12556 & 38515 & 0 \\
CONSENT & 33115 & 0.194 & 0.152 & 0.042 & 99.9 & 12509 & 38318 & 38 \\
Racon & 32183 & 0.276 & 0.190 & 0.085 & 99.2 & 12514 & 38421 & 72 \\
Canu & 25924 & 0.308 & 0.183 & 0.124 & 99.9 & 13280 & 38517 & 3 \\
Daccord & 31403 & 0.423 & 0.412 & 0.011 & 99.2 & 12604 & 38598 & 3 \\
\hline Ploidy=3 & & & & & & & & \\
\hline VeChat & 48085 & 0.031 & 0.015 & 0.016 & 100.0 & 12595 & 38467 & 13 \\
CONSENT & 50462 & 0.276 & 0.205 & 0.071 & 100.0 & 12511 & 38187 & 105 \\
Racon & 48986 & 0.558 & 0.427 & 0.131 & 98.7 & 12484 & 38257 & 288 \\
Canu & 37210 & 0.612 & 0.405 & 0.207 & 99.9 & 13675 & 38360 & 30 \\
Daccord & 48189 & 0.807 & 0.752 & 0.055 & 99.7 & 12485 & 38357 & 16 \\
\hline Ploidy=4 & & & & & & & & \\
\hline VeChat & 62743 & 0.074 & 0.047 & 0.027 & 99.9 & 12593 & 38442 & 44 \\
CONSENT & 66315 & 0.275 & 0.180 & 0.095 & 100.0 & 12492 & 38268 & 111 \\
Racon & 64342 & 0.547 & 0.398 & 0.149 & 93.2 & 12464 & 38016 & 387 \\
Canu & 46698 & 0.549 & 0.335 & 0.214 & 99.5 & 13956 & 38255 & 86 \\
Daccord & 63440 & 0.833 & 0.790 & 0.043 & 97.5 & 12463 & 38335 & 22 \\
\hline
\end{tabular}

Table 1. Error correction benchmarking results for simulated PacBio CLR reads of various polyploid genomes (ploidy $=2,3,4$ ). The average sequencing coverage per haplotype is $30 \mathrm{x}$ and sequencing error rate is $10 \%$. '\#Seq' indicates the number of corrected reads. The error rate is equal to the sum of mismatch and indel rate. The results are sorted by the error rate in ascending order. 


\begin{tabular}{lcccccccc}
\hline Method & \#Seq & $\begin{array}{c}\text { Error } \\
\text { rate } \\
(\%)\end{array}$ & $\begin{array}{c}\text { Mismatch } \\
(\%)\end{array}$ & $\begin{array}{c}\text { Indel } \\
(\%)\end{array}$ & $\begin{array}{c}\text { Haplotype } \\
\text { coverage } \\
(\%)\end{array}$ & $\begin{array}{c}\text { N50 } \\
(\mathrm{bp})\end{array}$ & $\begin{array}{c}\text { NGA50 } \\
(\mathrm{bp})\end{array}$ & $\begin{array}{c}\text { \# Mis- } \\
\text { assem- } \\
\text { blies }\end{array}$ \\
\hline Ploidy=2 & & & & & & & & \\
\hline VeChat & 30920 & 0.022 & 0.007 & 0.014 & 99.9 & 13095 & 40612 & 5 \\
CONSENT & 32661 & 0.212 & 0.160 & 0.052 & 99.9 & 13040 & 40903 & 37 \\
Racon & 31840 & 0.346 & 0.234 & 0.112 & 99.3 & 13039 & 40725 & 120 \\
Canu & 25506 & 0.390 & 0.206 & 0.183 & 100.0 & 13820 & 40846 & 6 \\
Daccord & 31438 & 0.438 & 0.410 & 0.027 & 99.2 & 12987 & 40730 & 3 \\
\hline Ploidy=3 & & & & & & & & 13130 \\
\hline VeChat & 45113 & 0.090 & 0.041 & 0.050 & 100.0 & 40497 & 90 \\
CONSENT & 49826 & 0.298 & 0.221 & 0.077 & 99.9 & 13037 & 40877 & 103 \\
Racon & 48520 & 0.673 & 0.501 & 0.172 & 98.6 & 13028 & 39286 & 631 \\
Canu & 36962 & 0.748 & 0.453 & 0.295 & 99.9 & 14141 & 40605 & 59 \\
Daccord & 49033 & 0.821 & 0.751 & 0.069 & 99.7 & 12712 & 39193 & 11 \\
\hline Ploidy=4 & & & & & & & & \\
\hline VeChat & 58739 & 0.169 & 0.098 & 0.071 & 99.7 & 13129 & 40450 & 177 \\
CONSENT & 65384 & 0.292 & 0.195 & 0.097 & 100.0 & 13033 & 40864 & 121 \\
Racon & 63670 & 0.666 & 0.469 & 0.197 & 94.9 & 13012 & 40007 & 668 \\
Daccord & 65072 & 0.840 & 0.784 & 0.056 & 96.9 & 12606 & 39076 & 22 \\
\hline
\end{tabular}

Table 2. Error correction benchmarking results for simulated Oxford Nanopore reads of various polyploid genomes (ploidy $=2,3,4$ ). The average sequencing coverage per haplotype is $30 \mathrm{x}$ and sequencing error rate is $10 \%$.

\begin{tabular}{lcccccccc}
\hline Method & \#Seq & $\begin{array}{c}\text { Error } \\
\text { rate } \\
(\%)\end{array}$ & $\begin{array}{c}\text { Mismatch } \\
(\%)\end{array}$ & $\begin{array}{c}\text { Indel } \\
(\%)\end{array}$ & $\begin{array}{c}\text { Haplotype } \\
\text { coverage } \\
(\%)\end{array}$ & $\begin{array}{c}\text { N50 } \\
(\mathrm{bp})\end{array}$ & $\begin{array}{c}\text { NGA50 } \\
(\mathrm{bp})\end{array}$ & $\begin{array}{c}\text { \# Mis- } \\
\text { assem- } \\
\text { blies }\end{array}$ \\
\hline \multicolumn{2}{l}{ Low complexity (20 genomes) } \\
\hline VeChat & 293466 & 0.036 & 0.020 & 0.015 & 96.9 & 11866 & 29555 & 104 \\
Racon & 299053 & 0.200 & 0.122 & 0.078 & 91.7 & 11811 & 29514 & 794 \\
CONSENT & 299333 & 0.214 & 0.149 & 0.065 & 98.4 & 11841 & 29556 & 515 \\
Canu & 253381 & 0.259 & 0.134 & 0.125 & 97.4 & 12370 & 29457 & 139 \\
Daccord & 298284 & 0.259 & 0.243 & 0.016 & 92.8 & 11862 & 29595 & 280 \\
\hline High complexity (100 genomes) $)$ & & & & & \\
\hline VeChat & 1441190 & 0.088 & 0.061 & 0.026 & 97.5 & 11886 & 30129 & 2774 \\
CONSENT & 1497216 & 0.274 & 0.163 & 0.112 & 99.4 & 11839 & 30204 & 3263 \\
Canu & 1185152 & 0.354 & 0.192 & 0.162 & 99.0 & 12706 & 30016 & 873 \\
Racon & - & - & - & - & - & - & - & - \\
Daccord & - & - & - & - & - & - & - & - \\
\hline
\end{tabular}

Table 3. Error correction benchmarking results for simulated PacBio CLR reads of metagenomic datasets with different complexity. The average sequencing coverage of strains is about $30 \mathrm{x}$ and the sequencing error rate is $10 \%$. 
Real sequencing data (Mock communities). Table 4 shows the error correction benchmarking results for real sequencing data. The three sections of the table show results on the yeast pseudo-diploid genome dataset (mock community) first, the NWC metagenome dataset (real) second, and the Microbial 10-plex metagenome dataset (mock community) as the third section of rows in Table ??. VeChat achieves approximately $2 \sim 4,1.4 \sim 7.8$ and $3.3 \sim 5.6$ times lower error rates on Yeast, NWC and Microbial 10-plex datasets, respectively, while maintaining comparable performance in terms of other aspects.

\begin{tabular}{|c|c|c|c|c|c|c|c|c|}
\hline Method & \#Seq & $\begin{array}{l}\text { Error } \\
\text { rate } \\
(\%)\end{array}$ & $\begin{array}{c}\text { Mismatch } \\
(\%)\end{array}$ & $\begin{array}{c}\text { Indel } \\
(\%)\end{array}$ & $\begin{array}{c}\text { Haplotype } \\
\text { coverage } \\
(\%)\end{array}$ & $\begin{array}{l}\text { N50 } \\
\text { (bp) }\end{array}$ & $\begin{array}{l}\text { NGA50 } \\
\text { (bp) }\end{array}$ & $\begin{array}{c}\text { \# Mis- } \\
\text { assem- } \\
\text { blies }\end{array}$ \\
\hline \multicolumn{9}{|c|}{ Yeast pseudo-diploid genome } \\
\hline VeChat & 107210 & 0.236 & 0.111 & 0.126 & 99.6 & 5693 & 15537 & 505 \\
\hline Daccord & 149020 & 0.503 & 0.285 & 0.217 & 96.5 & 4762 & 15161 & 1457 \\
\hline Racon & 136199 & 0.758 & 0.282 & 0.476 & 98.3 & 6349 & 16001 & 3836 \\
\hline Canu & 118367 & 0.787 & 0.214 & 0.573 & 99.9 & 5684 & 15603 & 743 \\
\hline CONSENT & 160136 & 0.947 & 0.344 & 0.603 & 99.4 & 5622 & 16001 & 7973 \\
\hline \multicolumn{9}{|c|}{ NWC metagenome } \\
\hline VeChat & 156426 & 0.101 & 0.031 & 0.070 & 99.3 & 9619 & 27416 & 14961 \\
\hline Daccord & 163313 & 0.140 & 0.079 & 0.061 & 99.5 & 8461 & 26343 & 12440 \\
\hline Racon & 168879 & 0.394 & 0.062 & 0.332 & 99.5 & 9914 & 28428 & 10832 \\
\hline Canu & 37779 & 0.551 & 0.090 & 0.461 & 99.1 & 13811 & 27729 & 4066 \\
\hline CONSENT & 176764 & 0.787 & 0.107 & 0.680 & 99.7 & 9708 & 27638 & 9731 \\
\hline \multicolumn{9}{|c|}{ Microbial 10-plex metagenome } \\
\hline VeChat & 245804 & 0.089 & 0.066 & 0.023 & 99.3 & 7837 & 17511 & 1533 \\
\hline Racon & 253817 & 0.297 & 0.160 & 0.137 & 97.8 & 8019 & 17760 & 3724 \\
\hline Canu & 193810 & 0.328 & 0.121 & 0.206 & 99.8 & 8477 & 17824 & 1170 \\
\hline Daccord & 254003 & 0.336 & 0.298 & 0.038 & 98.2 & 7704 & 17342 & 2073 \\
\hline CONSENT & 256935 & 0.495 & 0.107 & 0.388 & 100.0 & 8017 & 17729 & 3470 \\
\hline
\end{tabular}

Table 4. Error correction benchmarking results for real sequencing data (mock communities).

\section{Varying read coverage}

In order to evaluate how sequencing coverage influences the error correction approaches, we focuse on the triploid genome, consisting of three E. coli strains as described before. We simulated PacBio CLR reads at varying sequencing coverage, namely, 10x, 20x, 30x, 40x, 50x per haplotype.

Supplementary Table S2 shows the benchmarking results of error correction. In summary, VeChat achieves approximately $2 \sim 47$ times lower error rates on all datasets, while maintaining better or comparable performance in terms of other aspects such as number of corrected reads, completeness (HC) and length of corrected reads. As the sequencing coverage increases (from 10x to 50x), VeChat achieves better error correction (error rate from $0.311 \%$ to $0.017 \%$ ), while keeping comparable performance in terms of other aspects.

\section{Varying sequencing error rates}

In order to evaluate the effect of sequencing error rate on the different methods, we again focused on the triploid genome consisting of three E. coli strains as described above. Accordingly, we simulated PacBio CLR reads at varying sequencing error rates, namely, at 5\%,10\% and 15\%. The average sequencing coverage per haplotype is 30x.

Supplementary Table S3 shows the corresponding benchmarking results of error correction. Overall, VeChat achieves approximately $10 \sim 93,9 \sim 26$ and $7 \sim 9$ times lower error rates on datasets with $5 \%$, 
$10 \%$ and $15 \%$ errors, respectively, while maintaining comparable performance in terms of other aspects.

On decreasing sequencing error rate (from $15 \%$ down to $5 \%$ ), VeChat's error correction undoubtedly improves with the error rate achieved dropping from $0.091 \%$ to $0.009 \%$.

\section{Runtime and memory usage evaluation}

The runtime of VeChat is dominated by three steps: while the computation of read overlaps (without baselevel alignment) is extremely fast, subsequent edit-distance-based alignment (for segmenting windows) is time-consuming. Second, the POA algorithm that drives the construction of the variation graphs performs sequence to graph alignment, which comes at computational complexity of $O\left(N\left(2 N_{p}+1\right)|V|\right)$, where $N$ is the length of the sequence to be aligned, $N_{p}$ is the average number of predecessors in the graph and $|V|$ is the number of vertices in the graph (Lee et al., 2002). Third, VeChat follows an iterative paradigm, such as read overlap computation (with base-level alignment) and error correction (consensus generation) steps during the second iteration; this also requires a considerable amount of running time.

We performed all benchmarking analyses on x86_64 GNU/Linux machines using 48 cores. The runtime and peak memory usage evaluations for different methods are reported in Supplementary Tables S4 7. VeChat takes $23 \sim 81 \mathrm{CPU}$ hours and $8 \sim 92 \mathrm{CPU}$ hours on simulated PacBio CLR and ONT reads from datasets reflecting varying ploidy (2, 3 or 4 , as usual), which is $1.1 \sim 6.2$ and $0.6 \sim 6.9$ times slower than other methods. At the same time, it requires higher peak memory usages (Supplementary Tables S4, S5). VeChat is $2.4 \sim 7.1$ times slower than other approaches on the simulated metagenomic dataset, and reaches higher peak memory usages (Supplementary Table S6). Note that the high complexity metagenomic dataset is too large to be processed by Racon and Daccord, whereas our approach is able to handle it. In addition, VeChat is $0.7 \sim 32$ times slower on real datasets, while requiring higher peak memory usages (Supplementary Table S7).

\section{Discussion}

We have presented VeChat, as an approach that performs haplotype-aware error correction for thirdgeneration sequencing (TGS) reads. To the best of our knowledge, VeChat is the first approach that explicitly addresses to preserve haplotype-specific variation already during the correction process, which improves over prior approaches in particular insofar as bias inducing consensus sequences can be avoided. Results have demonstrated the superiority of VeChat: in all benchmarking scenarios, VeChat suppresses error rates by at least a factor of 2 or 3 , if not, as is the case for the majority of scenarios, suppressing error rates by one to two orders of magnitude in comparison with the leading competitors. At the same time, VeChat preserves the haplotype identity of the reads, which means that after correction with VeChat, all reads contribute to the coverage of the haplotype they stemmed from. The most obvious interpretation of these results is that capturing haplotype structure already during error correction is not just beneficial, but perhaps even imperative, when seeking to remove all errors from TGS reads. For appropriately capturing haplotype-specific variants during the error correction step, we construct variation graphs from the noisy TGS reads directly. Note that direct construction of variation graphs from heavily erroneous reads is not standard. In fact, at first glance, it is even counterintuitive, because the seminal idea of variation graphs is to be constructed from true haplotype-specific, sufficiently long patches of sequence. Here, patches of sequence contain up to even $15 \%$ of errors.

So, the resulting graph contains a large amount of nodes and edges that reflect such errors. For identifying spurious nodes and edges, one then exploits that sequencing errors are randomly distributed, whereas variants tend to re-occur across different reads. In particular, edges that link spurious nodes (with true nodes or other spurious nodes) tend to be little covered by reads, because reads, unlike for true effects, do not tend to share errors. To systematically identify spurious edges as edges that are covered by too little reads, in a sound, principled way, we adopt two metrics from frequent itemset mining. While Support measures the relative coverage of edges in the graph, Confidence measures the association between the two nodes incident to the edge they share; if basic support or the association is too little, the edge and possibly resulting isolated nodes are removed. 
A particular effect of VeChat is to achieve drastic improvements in terms of mismatch rates; improvements on indel rates are also clearly evident in all scenarios, but usually not necessarily drastic. One possible explanation is that substitution events, much more than insertion and deletion events, dominate the evolutionary processes of living organisms, and thus are often characteristic of strains or haplotypes. VeChat appears to be the first approach to correctly preserve these single nucleotide polymorphisms (SNPs), because the distinction of haplotypes is just what variation graphs are made for. At any rate, VeChat appears to prevent masking of true variants as a result of generating consensus sequence.

As for future perspectives, the rapid development of long-read sequencing technologies will lead to decreasing sequencing error rates. However, because the advantages of VeChat become even more evident when sequencing error rates drop, VeChat will also be a superior tool when correcting long reads in which errors appear at a rate of $5 \%$ or lower (see Supplementary Table S3).

Of course, future improvements are conceivable: in particular, VeChat requires computational resources that exceed those of other approaches. In particular on large datasets, VeChat experiences longer runtimes and higher peak memory usage. However, there is room for improving on the computational efficiency of VeChat. One crucial point is that VeChat uses off-the-shelf approaches in some places. While these approaches do the job, not all features they provide are needed, which corresponds to an overhead of computations when running the off-the-shelf software. In particular, there is good hope that computations such as edit-distance-based alignments, or the sequence-to-graph alignments, can be replaced by more efficient routines in the future.

\section{Methods}

\section{Step 1: Read overlap calculation}

Step 1 refers to computation of all-vs-all overlaps for the input reads (first cycle: raw reads, second cycle: pre-corrected reads) using the (widely popular) minimizer based, long-read overlap computation tool Minimap2 (Li, 2018). During the first cycle, only a seed-chain procedure is performed, while during the second cycle a base-level alignment is added. Because this is extremely fast, it can easily manage the large amount of read pairs we need to process. Subsequently, bad overlaps are filtered by reasonable, additional criteria, which includes removing overlaps that do not exceed 500 bp in length, self-overlaps, or internal matches. In this, we follow Algorithm 5 in $(\mathrm{Li}, 2016)$ and its implementation in (Marijon et al., 2020). Additionally, overlaps that have a high error rate, that is $\left|1-\min \left(L_{q}, L_{t}\right) / \max \left(L_{q}, L_{t}\right)\right| \geq e$, where $L_{q}$ and $L_{t}$ are the overlap lengths of the query and target read, respectively, and $e$ is the maximum error-rate threshold, are also filtered out.

While during the second cycle, the similar procedures(overlap computation and filtration) are also performed but for pre-corrected reads. Unlike in the first cycle, we compute pre-corrected read overlaps with base-level alignment such that the sequence identity of overlaps (overlap identity) can be determined, and then filter overlaps with one more criterion, minimum overlap identity (denoted as $\delta, \delta=0.99$ for simulated sequencing data and $\delta=0.98$ for real sequencing data). Notably, because most of sequencing errors have been corrected in the first cycle, the identity distribution of read overlaps derived from different haplotypes is clearly dispersed. Therefore, it is straightforward to filter overlaps from different haplotypes by simply setting overlap identity $\geq \delta$.

\section{Step 2: Read alignment pile generation}

Our workflow then selects a target read $r$ and, based on the overlaps computed in step 1, collects reads that overlap $r$. The target read $r$ serves as a backbone, and for each overlap between read $r$ and another read, a fast edit-distance-based alignment (Myers, 1999; Šošić and Šikić, 2017) is then performed, which generates a read alignment pile. The edit-distance-based alignment is only needed to split the read alignment pile into small windows in step 3. Dangling ends of reads that overlap the target read $r$, 
indicated by horizontal dotted lines in the original read alignment pile in Figure 1, are removed from further consideration in the following. See (Vaser et al., 2017) for more details.

\section{Step 3: Window segmentation}

The read alignment pile is then divided into several small non-overlapping windows of identical length, with the target read serving as a reference: each such window covers $500 \mathrm{bp}$ of the target read. Obviously, segmenting the read alignment pile reflects a straightforward procedure, because of the pairwise alignments of the target read with its overlapping reads served as the basis for read alignment pile construction, see Step 2 (Vaser et al., 2017). The part of the target read $r$ corresponding with one particular window is further referred to as a 'target subread'. This implies in particular that target subreads are $500 \mathrm{bp}$ in length, apart from the rightmost window, where target subreads can be shorter,

The reason for segmenting the alignment piles into windows of small length is the great reduction in terms of computational burden in the following: the next step 4, as the technical core of our approach being concerned with variation graphs, greatly profits from this segmentation, both in terms of downsizing the original problem as well as in terms of enabling parallelization.

\section{Step 4: Error correction for target subreads}

Step 4 reflects the methodical novelty of VeChat. Step 4 differs when comparing the first with the second cycle, see Figure 2(a) (a) for the first and Figure 2(a) (b) for the second cycle. Step 4 of the first cycle is considerably more involved, because it reflects the crucial statistical considerations through which to identify sequencing errors. The core idea that underlies these crucial statistical considerations is that true variants are significantly likely to co-occur across different reads, whereas occurrence of errors is random. Correspondingly, the following arguments make sense.

Thanks to dividing reads into sub-reads, the corresponding computations, such as variation graph construction and statistical evaluation of edges relative to read coverage, can be parallelized across sub-reads, which speeds up computations considerably.

\section{Variation graph construction}

The subsequences in a window we refer to as subreads, are subsequently used to construct a variation graph $G=(V, E, P)$. This variation graph is a directed acyclic graph (DAG), where vertices $v \in V$ represent nucleotides $(\mathrm{A}, \mathrm{T}, \mathrm{C}, \mathrm{G})$, edges $\left(v_{i}, v_{j}\right) \in E$ indicate that the nucleotides represented by nodes $v_{i}$ and $v_{j}$ have appeared as a two-letter subsequence in one of the reads from which the graph was constructed, relative to that particular position with respect to the read alignment pile. So, for example, if $v_{i}$ and $v_{j}$ correspond to $A$ and $G$, respectively, exactly the reads that relative to the coordinates implied by the target read show $A G$ at that particular position induce an edge $\left(v_{i}, v_{j}\right)$. Correspondingly, reads can be identified as certain paths $P=\left(v_{1}, \ldots, v_{l}\right)$ in the variation graph. For variation graph construction, we use the partial order alignment (POA) algorithm (Lee et al., 2002) and its faster version, enhanced by SIMD vectorization, as described in (Vaser et al., 2017).

\section{Pruning: Principle}

In the following, we will use the notation $v \in V$ to also indicate the letter from the alphabet $\{A, C, G, T\}$ a particular node $v$ refers to.

The high error rate affecting TGS reads and the possible bias introduced by the POA algorithm (because, for example, the POA depends on the order relative to which reads are considered), the variation graph constructed in the first cycle contains many spurious vertices and edges.

For pruning the graph from mistaken edges and/or nodes (vertices), we adopt techniques from frequent itemset mining. The basic idea is to identify edges with itemsets, and to prune edges from the graph if the corresponding itemsets do not appear to be sufficiently frequent. After removal of edges, reads are re-aligned against the resulting graph, such that itemset counts have to be re-computed. 
This may render more edges to correspond to itemsets that are not sufficiently frequent. The cycle of identifying edges as infrequent itemsets, removing them, and re-aligning reads is repeated until convergence, that is, until no further edges are identified as to be removed. In practice, we determined 3 as an appropriate number of iterations for our experiments. Note that for re-aligning reads against the modified graph, we make use of the POA algorithm, without, however, re-modifying the graph anymore.

The model that underlies the mining of frequent itemsets is the "market-basket model". Baskets correspond to sets of items, and frequent itemsets correspond to subsets of items that appear in sufficiently many baskets, or, vice versa, infrequent itemsets correspond to subsets of items that do not appear in sufficiently many baskets.

Following this model, the basic set of items agrees with the set of nodes $V$ in the variation graph. Baskets then correspond to reads, which are modeled as paths $P=\left(v_{1}, \ldots, v_{l}\right)$, and the items they contain correspond to the nodes $v_{1}, \ldots, v_{l} \in V$ the reads cover. Further, the itemsets we are interested in correspond to edges $e=(v, w)$, as pairs of items $v, w$. If two consecutive nodes $v, w$, as particular subsets of items, do not appear in sufficiently many baskets, that is, are not contained in that particular order in sufficiently many paths $P$, the corresponding edge $e=(v, w)$ is removed from the graph.

For appropriately quantifying "sufficiently many baskets", we make further use of Support and Confidence as two standard definitions from frequent itemset mining. While "Support" just corresponds to the number of baskets a particular subset of items is contained in, "Confidence" corresponds to measuring whether appearance of items in basket is correlated (or, in other words, whether sets of items are "associated" with each other). Here, Support just agrees with the number of reads by which a particular edge $e=(v, w)$ is covered. Confidence corresponds to the amount of reads that cover the edge $(v, w)$ in relation to how many reads cover $v$, on the one hand, and in relation to how many reads cover $w$, on the other hand. If neither sufficiently many reads that cover $v$ also cover $(v, w)$, nor sufficiently many reads that cover $w$ also cover $(v, w)$, we "loose confidence" in $e=(v, w)$, because the edge $(v, w)$ could reflect sequencing error noise, and remove it.

\section{Pruning: Definitions}

To make the ideas from above explicit, let $R(v)$ be all reads that cover node $v$. For $r \in R(v)$, let further $p_{r, v}$ reflect the probability that $v$ reflects an error in $r$. When dealing with FASTQ files, the probability $p_{r, v}$ is derived from the Phred profile of $r$. In case of FASTA files, $p_{r, v}$ is taken as zero.

We now would like to determine $w(v)$, as a weight for node $v$ that reflects the expected number of reads that cover it. Note that for FASTA files, $w(v)$ just agrees with the number of reads that cover $v$. For FASTQ files, $w(v)$ corresponds to summing up $1-p_{r, v}$ across all reads $r \in R(v)$, as the sum of the probabilities that the reads $r \in R(v)$ indeed reflect the letter associated with $v$. In terms of formulas, we obtain

$$
w(v):= \begin{cases}\sum_{r \in R(v)} 1 & \text { for FASTA files } \\ \sum_{r \in R(v)} 1-p_{r, v} & \text { for FASTQ files }\end{cases}
$$

For an edge $e=\left(v_{i}, v_{j}\right)$, we further determine

$$
w(e)=w\left(v_{i}, v_{j}\right):=\frac{1}{2} \sum_{r \in R\left(v_{i}\right) \cap R\left(v_{j}\right)} w\left(v_{i}\right)+w\left(v_{j}\right)
$$

as an approximation for the expected amount of reads that cover $e=\left(v_{i}, v_{j}\right)$. Note that $w(e)$ corresponds to exactly the amount of reads that cover $e$ for FASTA files. For FASTQ files, the expected amount of reads that cover $\left(v_{i}, v_{j}\right)$ virtually corresponds to the sum of products $\left(1-p_{r, v_{i}}\right)\left(1-p_{r, v_{j}}\right)=$ $1-p_{r, v_{i}}-p_{r, v_{j}}+p_{r, v_{i}} p_{r, v_{j}}(*)$ across $r \in R\left(v_{i}\right) \cap R\left(v_{j}\right)$. Here, for speeding up computations, we opt for approximating $\left(^{*}\right)$ by $1-\frac{1}{2} p_{r, v_{i}}-\frac{1}{2} p_{r, v_{j}}$. Since this agrees with $(*)$ in terms of orders of magnitude, this introduces only negligible deviations from the true values; experiments of ours (data not shown) confirm that the gain in speed offsets the loss in precision on that account. 
Based on these weights, we now define the two metrics Support and Confidence. See also Figure 3 for an illustration. In formal detail, let $e=\left(v_{i}, v_{j}\right)$ be an edge. Let then Support of $e$ be defined as

$$
\operatorname{Support}(e):=w(e)
$$

that is, just as the weight of $e$. Further, note that Confidence is an asymmetrical measure: the probability to observe $v_{j}$ in a read that contains $v_{i}$ may disagree with the probability to observe $v_{i}$ in a read that contains $v_{j}$. We take this into account by defining

$$
\operatorname{Confidence}\left(v_{i}, v_{j}\right):=\operatorname{Confidence}\left(v_{i} \rightarrow\left\{v_{i}, v_{j}\right\}\right)=\frac{w\left(v_{i}, v_{j}\right)}{\sum_{v_{k^{\prime}} \in S\left(v_{i}\right)} w\left(v_{i}, v_{k}^{\prime}\right)}
$$

on the one hand, and

$$
\operatorname{Confidence}\left(v_{j}, v_{i}\right):=\operatorname{Confidence}\left(v_{j} \rightarrow\left\{v_{i}, v_{j}\right\}\right):=\frac{w\left(v_{i}, v_{j}\right)}{\sum_{v_{k} \in P\left(v_{j}\right)} w\left(v_{k}, v_{j}\right)}
$$

on the other hand, where $S\left(v_{i}\right)$ and $P\left(v_{j}\right)$ denote the vertices that succeed $v_{i}$ and precede $v_{j}$, respectively (and where $v_{i} \rightarrow\left\{v_{j}, v_{j}\right\}$ and $v_{j} \rightarrow\left\{v_{i}, v_{j}\right\}$ agree with standard notation from association rule mining). We eventually declare

$$
\text { Confidence }(e):=\max \left\{\operatorname{Confidence}\left(v_{i}, v_{j}\right) \text {, Confidence }\left(v_{j}, v_{i}\right)\right\}
$$

as the overall Confidence in $e=\left(v_{i}, v_{j}\right)$.

It remains to determine appropriate thresholds $s$ and $c$, such that edges $e$ for which either $\operatorname{Support}(e)<$ $s$ or Confidence $(e):<c$ are pruned from the graph. Note that by its definition, Confidence reflects the probability that a read that covers $v_{i}$ also covers $v_{j}$, or vice versa. We determined $c=0.2$ as an appropriate threshold in experiments; see the Supplement for the corresponding experiments.

Support, however, does not reflect a probability. Depending on the overall amount of reads in a subwindow, and the length of a subwindow - that is virtually depending on the average read coverage of a subwindow) - the Support needs to be appropriately scaled. Therefore, consider that $C:=\sum_{v \in V} w(v) / L$ is the average coverage of a position in the subwindow. Accordingly, we determine $s:=0.2 \times C$ as a threshold that takes subwindow specific coverage into appropriate account.

Note eventually that both Support and Confidence are required for effective pruning of the graph, see Figure S2 for the correlation between the two quantities.

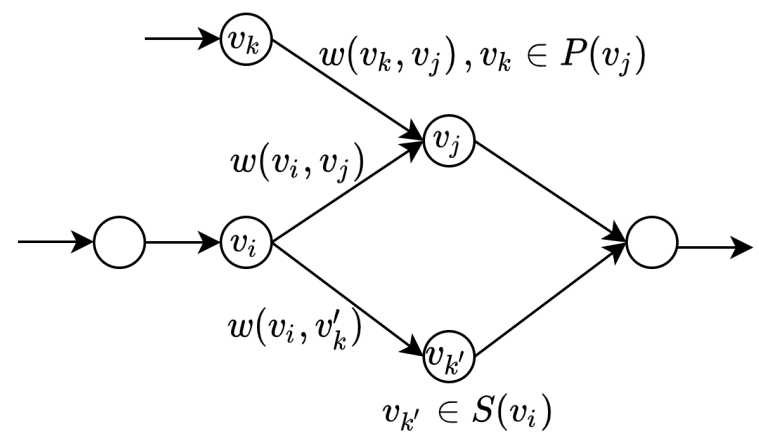

Figure 3. A schematic diagram for explaining the calculation of Support and Confidence for edge $\left(v_{i}, v_{j}\right)$.

\section{Optimal alignment path extraction}

Upon convergence of the pruning algorithm, the target subread that corresponds to a small window is realigned against the fully pruned variation graph that results from the last iteration of the pruning algorithm. The path in the graph that corresponds to the optimal alignment of the target subread is then taken as the pre-corrected target subread; see the orange elements in Figure 2(a) for an illustration. 


\section{Step 5: Concatenation}

In this step, pre-corrected target subreads are concatenated to a whole, pre-corrected target read, which corresponds to the obvious, straightfoward idea of "patching together" pre-corrected target subreads; see "5. Concatenation" in Figure 1 for an illustration.

\section{Step 6: Merging corrected target reads}

Steps 2-5 are repeated until all reads have been corrected. The overall set of pre-corrected that results from these steps are then taken as input for the second cycle.

\section{Second Cycle: Modifications Step 1 and 4}

Note that the pre-corrected reads generated by way of cycle 1 still contain a small, but yet non-negligible amount of random errors. Cycle 2 addresses to correct these remaining errors. To do so, steps 1-6 are repeated with, however, some crucial modifications in steps 1 and 4. See the blue elements in Figure 1 for the workflow that reflects the procedures of cycle 2 . To be specific, the modification of step 1 consists in not only computing all-vs-all overlaps based on minimizers, but also base-level alignments (Li, 2018) for the pre-corrected reads. This considerably facilitates to filter reads overlaps according to which they stem from identical haplotypes, based on sequence identity related thresholds. We use $\delta=0.99$ for sequencing error rates of $5-10 \%$ and $\delta=0.98$ for a sequencing error rate of $15 \%$ in our experiments, which we also generally recommend.

The modification of step 4 then relates to generating a single consensus sequence from each variation graph instead of performing iterative graph pruning and sequence-to-graph re-alignment. For that, the dynamic programming algorithm (called "heaviest bundle algorithm" because the traversal algorithm selects the path by maximum weight) (Lee, 2003) as indicated in Figure 2(b) is used. Note that generating consensus sequences from graphs is reasonable in the second cycle, because the overlapping reads used to correct the target read stem from the same haplotype. Finally, we obtain fully error-corrected reads as the output of the second cycle; see "All final corrected reads (cycle2)" in Figure 1.

\section{Acknowledgements}

Not applicable.

\section{Funding}

XL and XK were supported by the Chinese Scholarship Council. AS was supported by the Dutch Scientific Organization, through Vidi grant 639.072.309 during the early stages of the project, and from the European Union's Horizon 2020 research and innovation programme under Marie Skłodowska-Curie grant agreements No 956229 (ALPACA) and No 872539 (PANGAIA).

\section{Availability of data and materials}

Raw sequencing data can be downloaded from Zenodo (DOI: 10.5281/zenodo.5501454). The results can be reproduced from Code Ocean (DOI: 10.24433/CO.2329278.v2). The source code of VeChat is GPL-3.0 licensed, and publicly available at https://github.com/HaploKit/vechat.

\section{Ethics approval and consent to participate}

Not applicable. 


\section{Competing interests}

The authors declare that they have no competing interests.

\section{Consent for publication}

Not applicable.

\section{Authors' contributions}

XL and AS developed the method. XL implemented the software and conducted the data analysis. XK simulated the metagenomic data sets. XL and AS wrote the manuscript. All authors read and approved the final version of the manuscript.

\section{References}

Bao, E. et al (2019). Flas: fast and high-throughput algorithm for pacbio long-read self-correction. Bioinformatics, 35(20), $3953-3960$.

Edge, P. and Bansal, V. (2019). Longshot enables accurate variant calling in diploid genomes from single-molecule long read sequencing. Nature communications, 10(1), 1-10.

Firtina, C. et al (2018). Hercules: a profile hmm-based hybrid error correction algorithm for long reads. Nucleic acids research, 46(21), e125-e125.

Fritz, A. et al (2019). Camisim: simulating metagenomes and microbial communities. Microbiome, 7(1), 1-12.

Fujimoto, A. et al (2021). Whole-genome sequencing with long reads reveals complex structure and origin of structural variation in human genetic variations and somatic mutations in cancer. Genome medicine, 13(1), 1-15.

Hackl, T. et al (2014). proovread: large-scale high-accuracy pacbio correction through iterative short read consensus. Bioinformatics, 30(21), 3004-3011.

Jain, C. et al (2018a). High throughput ani analysis of 90k prokaryotic genomes reveals clear species boundaries. Nature communications, $\mathbf{9}(1), 1-8$.

Jain, M. et al (2018b). Nanopore sequencing and assembly of a human genome with ultra-long reads. Nature biotechnology, 36(4), $338-345$.

Kolmogorov, M. et al (2020). metaflye: scalable long-read metagenome assembly using repeat graphs. Nature Methods, 17(11), 1103-1110.

Koren, S. et al (2017). Canu: scalable and accurate long-read assembly via adaptive k-mer weighting and repeat separation. Genome research, $\mathbf{2 7}(5), 722-736$.

Lee, C. (2003). Generating consensus sequences from partial order multiple sequence alignment graphs. Bioinformatics, 19(8), 999-1008.

Lee, C., Grasso, C. and Sharlow, M.F. (2002). Multiple sequence alignment using partial order graphs. Bioinformatics, 18(3), $452-464$.

Li, H. (2016). Minimap and miniasm: fast mapping and de novo assembly for noisy long sequences. Bioinformatics, 32(14), $2103-2110$.

Li, H. (2018). Minimap2: pairwise alignment for nucleotide sequences. Bioinformatics, 34(18), 3094-3100.

Logsdon, G.A., Vollger, M.R. and Eichler, E.E. (2020). Long-read human genome sequencing and its applications. Nature Reviews Genetics, 21(10), 597-614.

Luo, X., Kang, X. and Schönhuth, A. (2021). phasebook: haplotype-aware de novo assembly of diploid genomes from long reads. Genome biology, 22(1), 299.

Marijon, P., Chikhi, R. and Varré, J.S. (2020). yacrd and fpa: upstream tools for long-read genome assembly. Bioinformatics, 36(12), 3894-3896. 
Miga, K.H. et al (2020). Telomere-to-telomere assembly of a complete human x chromosome. Nature, 585(7823), 79-84.

Mikheenko, A. et al (2018). Versatile genome assembly evaluation with quast-lg. Bioinformatics, 34(13), i142-i150.

Morisse, P., Lecroq, T. and Lefebvre, A. (2018). Hybrid correction of highly noisy long reads using a variable-order de bruijn graph. Bioinformatics, 34(24), 4213-4222.

Morisse, P. et al (2021). Scalable long read self-correction and assembly polishing with multiple sequence alignment. Scientific reports, 11(1), 1-13.

Myers, G. (1999). A fast bit-vector algorithm for approximate string matching based on dynamic programming. Journal of the $A C M(J A C M)$, 46(3), 395-415.

Ono, Y., Asai, K. and Hamada, M. (2021). Pbsim2: a simulator for long-read sequencers with a novel generative model of quality scores. Bioinformatics, 37(5), 589-595.

Quince, C. et al (2017). Desman: a new tool for de novo extraction of strains from metagenomes. Genome biology, 18(1), 1-22.

Ruan, J. and Li, H. (2020). Fast and accurate long-read assembly with wtdbg2. Nature methods, 17(2), 155-158.

Salmela, L. and Rivals, E. (2014). Lordec: accurate and efficient long read error correction. Bioinformatics, 30(24), 3506-3514.

Salmela, L. et al (2017). Accurate self-correction of errors in long reads using de bruijn graphs. Bioinformatics, 33(6), 799-806.

Schrinner, S.D. et al (2020). Haplotype threading: accurate polyploid phasing from long reads. Genome biology, 21(1), 1-22.

Shafin, K. et al (2020). Nanopore sequencing and the shasta toolkit enable efficient de novo assembly of eleven human genomes. Nature Biotechnology, pages 1-10.

Somerville, V. et al (2019). Long-read based de novo assembly of low-complexity metagenome samples results in finished genomes and reveals insights into strain diversity and an active phage system. BMC microbiology, 19(1), 1-18.

Šošić, M. and Šikić, M. (2017). Edlib: a c/c++ library for fast, exact sequence alignment using edit distance. Bioinformatics, 33(9), 1394-1395

Thibodeau, M.L. et al (2020). Improved structural variant interpretation for hereditary cancer susceptibility using long-read sequencing. Genetics in Medicine, 22(11), 1892-1897.

Tischler, G. and Myers, E.W. (2017). Non hybrid long read consensus using local de bruijn graph assembly. bioRxiv, page 106252 .

Vaser, R. et al (2017). Fast and accurate de novo genome assembly from long uncorrected reads. Genome research, 27(5), 737-746. 